\title{
Design of advanced airfoil for stall-regulated wind turbines
}

\author{
Francesco Grasso $^{1}$, Domenico Coiro ${ }^{2}$, Nadia Bizzarrini ${ }^{2}$, and Giuseppe Calise ${ }^{2}$ \\ ${ }^{1}$ Aerodynamix, Naples, 80128, Italy \\ ${ }^{2}$ Dip. Ingegneria Industriale, Università di Napoli FedericoII, Naples, 80123, Italy \\ Correspondence to: Francesco Grasso (skyflash@inwind.it)
}

Received: 19 January 2017 - Discussion started: 1 February 2017

Revised: 22 May 2017 - Accepted: 30 June 2017 - Published: 27 July 2017

\begin{abstract}
Nowadays, all the modern megawatt-class wind turbines make use of pitch control to optimise the rotor performance and control the turbine. However, for kilowatt-range machines, stall-regulated solutions are still attractive and largely used for their simplicity and robustness. In the design phase, the aerodynamics plays a crucial role, especially concerning the selection/design of the necessary airfoils. This is because the airfoil performance is supposed to guarantee high wind turbine performance but also the necessary machine control capabilities. In the present work, the design of a new airfoil dedicated to stall machines is discussed. The design strategy makes use of a numerical optimisation scheme, where a gradient-based algorithm is coupled with the RFOIL code and an original Bezier-curves-based parameterisation to describe the airfoil shape. The performances of the new airfoil are compared in free- and fixed-transition conditions. In addition, the performance of the rotor is analysed, comparing the impact of the new geometry with alternative candidates. The results show that the new airfoil offers better performance and control than existing candidates do.
\end{abstract}

\section{Introduction}

Looking back in wind turbine history, pitch-regulated machines gradually substituted stall-regulated systems. In fact, the possibility to optimise the power production for each wind condition by regulating the pitch angle of the blade, proved to be a key feature to maximise the annual energy production (AEP) of the wind turbines. Nowadays, all the modern megawatt-class wind turbines are by default pitchregulated and several innovations are implemented by industry to improve the pitch performance (e.g. individual pitch control, fine regulation mechanisms/algorithms) and extract more power.

In apparent contradiction with megawatt machines, however, small and medium kilowatt wind turbines are still largely stall-regulated machines. The reasons for this are easy to give. The advantages of the pitch system in fact come at some costs. The first is the literal cost of the pitch system and its maintenance. Secondly, the pitch system increases the general complexity of the system, together with the development costs and the issues related to the system robustness/reliability. Extra components, such as onboard anemometers and pitch bearings are necessary to operate the pitch of the blade correctly. All these costs and complications can be very relevant for small machines, and it explains why a robust and easy-to-maintain solution is preferred even with some AEP sacrifice.

From the design point of view, the stall-regulated machines still offer a challenging task, especially concerning the aerodynamics of the blade that is supposed to ensure the power performance but also provide the machine control. In practice, the design of the blade should obviously aim to maximise the AEP, but it is also the only component to keep the turbine under control, stopping it when necessary. To do so, the stall and post-stall characteristics of the airfoils play a crucial role. From this angle, the selection/design of the airfoils and the blade shape design are more delicate than in pitch-regulated turbines.

The present work focuses on the design of a new airfoil specifically designed for stall-regulated turbines. The next section illustrates the design of the new airfoil in compari- 
son with existing geometries. Then, its impact on the overall turbine performance is discussed.

\section{Design of the new airfoil}

\subsection{General requirements}

The selection of the proper airfoils is very relevant to achieve satisfactory wind turbine performance. Depending on the area of the blade, the requirements change quite a lot; in fact, the outer sections are optimised for high aerodynamic performance, while the inner sections are designed to provide a low weight and structural integrity for the blade.

The focus of the present investigation is the outer region of the blade, so the airfoils should have high aerodynamic efficiency $(L / D)$. This is the primary parameter to increase the annual energy production of the rotor, but it is not the only one. Besides that, the stall behaviour should be considered, avoiding sharp stall. This would lead to load problems with the blade (e.g. fatigue issues and additional noise) and other components. The impact of roughness on the rotor performance should be also addressed when the airfoil is designed/selected. Normally, the annual production decreases when the blade is affected by dirt (e.g. mosquitos), damage (e.g. erosion) or imperfections. Designing an airfoil that is robust (or less sensitive) to roughness would contribute to maintaining a stable performance in the long run. Thus, it is important to have airfoils with a reduced drop in maximum lift coefficient and aerodynamic efficiency in rough conditions. In addition, limited variations in terms of corresponding angles of attack are desirable.

Regarding the blade construction, it must be buildable and lightweight to save production costs, so the airfoils adopted should not have critical features which may compromise those aspects (e.g. too thin trailing edge, very concave complex areas). Inevitably, there is interaction between weight minimisation and annual energy production optimisation, where the first would lead, for instance, to a large thickness distribution to accommodate a structurally efficient spar and maximise the section's moment of inertia, while the second would tend to reduce the airfoil thickness to reduce the drag.

A complete discussion can be found in Grasso (2011).

\subsection{Airfoils for stall-regulated wind turbines}

In addition to what has been presented in the previous paragraph, special considerations should address the peculiarity of stall-regulated wind turbines. As mentioned, the big challenge of these machines is their control. While the pitchregulated turbines can change the pitch angle of the blades, to optimise the performance for each wind speed, the stallregulated turbines are much simpler and rely only on the aerodynamics of the airfoils. This increases the complexity of the airfoil design.
First of all, the airfoils of stall-regulated turbines work in quite a wide range of angles of attack, so a sound performance comes from the fact that they achieve high aerodynamic efficiency over the angle of attack range. This is an important element to properly set up the design process. In fact, a design point close to stall would be desirable to obtain the best AEP performance, and the margin must be carefully calibrated and reduced compared to the values for pitch-regulated machines. The stall mechanism stops the turbine when the loads are becoming too large; postponing the stall could lead to excessive forces on the blades and the other components of the turbine. Furthermore, the capability to control the machine, slowing down the rotor and avoiding over-power issues depends on the airfoil stall and poststall behaviour. In fact, a slope of the lift curve that is excessively "flat" could be insufficient to control the turbine (and so prevent over-power), while a sharp stall would make it more difficult to re-start the machine and would cause sudden changes in the loads faced by the blades. In addition to this, the airfoil post-stall response is fundamental to avoid stall-induced vibrations, which is one of the main issues to address in designing stall-regulated machines.

\subsection{The stall-induced vibration phenomenon and its impact on airfoil design}

When a wind turbine blade vibrates, the aerodynamic forces have an additional component originating in the vibration velocity. Such a component can, with good approximation, be considered proportional to the vibration velocity; thus, it actually acts as a viscous damping force, usually denoted as "aerodynamic damping" (see Petersen et al., 1998; Rasmussen at al., 1993; Rasmussen, 1994). When the airfoils are in stall conditions, the slope of the lift curve becomes negative and can cause a local negative aerodynamic damping in the lift direction.

As an example, a descending airfoil will see an increasing angle of attack that will cause a lower value of lift coefficient; this will be equivalent to having a component of the aerodynamic force promoting the descent of the airfoil, thus acting as a negative damping force.

If global aerodynamic damping of the blade is both negative and larger (in magnitude) than the structural damping, any disturbance can cause divergent oscillations which can dramatically increase fatigue loads and can even lead to rapid failure in the worst case.

This phenomenon is usually referred to as "stall-induced vibrations" and represents a key issue for stall-regulated wind turbines, which work in stalled conditions for a significant part of their lifetime.

Stall-induced vibrations have to be regarded as instabilities of the blades that can take place due to any initial disturbance. A sharp stall leads to a lower damping force and so to larger vibrations. On the other hand, a flat lift curve beyond the stall could be insufficient to control the turbine. 


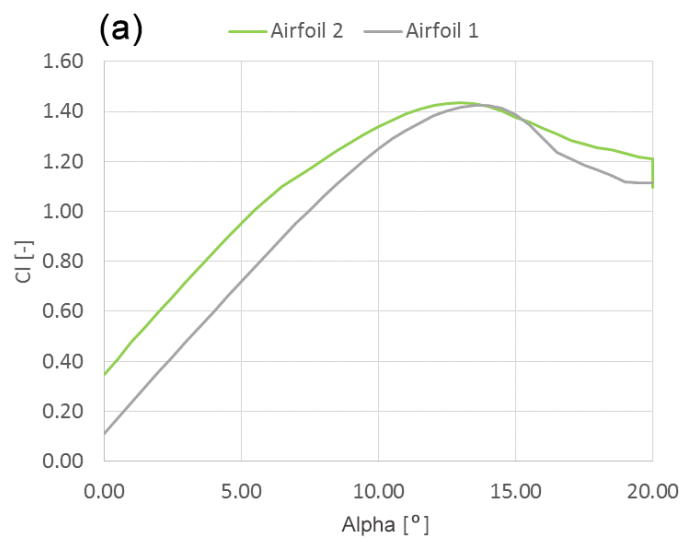

(b)
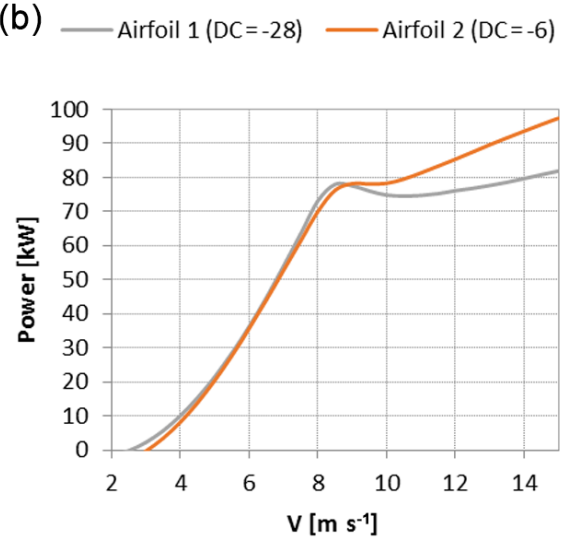

Figure 1. Power curve (b) generated as effect of different airfoil stall behaviour (a). The damping coefficient for both cases is indicated.

Low stall-induced vibrations and power control represent two conflicting requirements which make the design of a stall-regulated wind turbine a highly complex challenge. Finding a good compromise between these two aspects has been one of the main efforts in this work.

During the preliminary design phase, a simplified expression of the aerodynamic damping of the blade has been used to predict the dynamic behaviour of the blades without the need of any aeroelastic analysis, to make the design as fast as possible.

The linearised approach presented by Petersen et al. (1998) has been applied to obtain a simplified expression for the local aerodynamic damping on the different sections of the blades, using only quasi-steady, 2-D aerodynamics of the airfoils. Then, a simplified modal approach has been implemented to evaluate the aerodynamic damping of the complete blade, obtaining a damping coefficient (DC) used as an index of eventual oscillation amplitude. The use of this damping coefficient has been validated with several cases of wind turbines obtained during the optimisation process, giving always results coherent with the behaviour of the blades evaluated through aeroelastic analysis.

From the expression of the local damping coefficient in the out-of-plane direction (that usually is very close to the flapwise direction), it is possible to see that a gentle stall of the airfoils along the blade (which means a small value of the absolute value $\left|\frac{\mathrm{dCl}}{\mathrm{d} \alpha}\right|$ beyond the stall) would be desirable to avoid the occurrence of stall-induced vibrations. The expression of modal damping coefficients (both in edge-wise and in flap-wise directions) provides another useful information for the optimisation process. For each direction and for each mode, the modal aerodynamic damping coefficient can be interpreted as a linear combination of the local damping coefficients of the different sections along the blade, each one multiplied by the local displacement related to the mode shape. Looking at typical mode shapes of a wind turbine blade, considered to be a cantilevered beam, it can be observed that the highest displacements always occur on the outer part of the blade. This means that the largest contribution to the damping of the blade is given by the outer sections. Thus, the blade optimisation to avoid stall-induced vibrations can be limited at this part of the blade.

The typical effect of using an airfoil with a smoother stall in the outer half of the blade is shown in Fig. 1, in terms of power curve and modal aerodynamic DC. It can be seen how a gentle slope of lift coefficient curve of the airfoils (Airfoil 2) results in a reduction in the absolute value of DC with the related stall-induced vibrations but in less power control at high wind speeds.

So overall, it is important that the stall margin is reduced, but with gentle and continuous stall. To limit the problem of power control, the airfoils along the blade should have a low lift coefficient beyond stall and a drag coefficient that is as high as possible.

To meet the challenging scenario, these characteristics must be achieved both in clean and rough conditions. This introduces more complexity for the designer. In fact, special attention should be paid to ensuring that the characteristics of the lift curve do not change significantly with regard to stall and post-stall behaviour.

During the rotor design, the "rough" power curve is considered because it is the most conservative in terms of overall performances and power control. The "clean" power curve is considered because it is the most conservative for extreme and fatigue loads (due to higher stall-induced vibrations caused by a more abrupt stall).

\subsection{Design methodology}

Multidisciplinary design optimisation (MDO) (see Fletcher, 1987) has been adopted in this work. In fact, when compared to a traditional design technique (e.g. inverse design), MDO leads to a more accurate and computational time-saving design product, while covering constraints coming from different disciplines. Based on the authors' experience (see Bizzarrini et al., 2011; Grasso, 2012), a gradient-based algorithm 


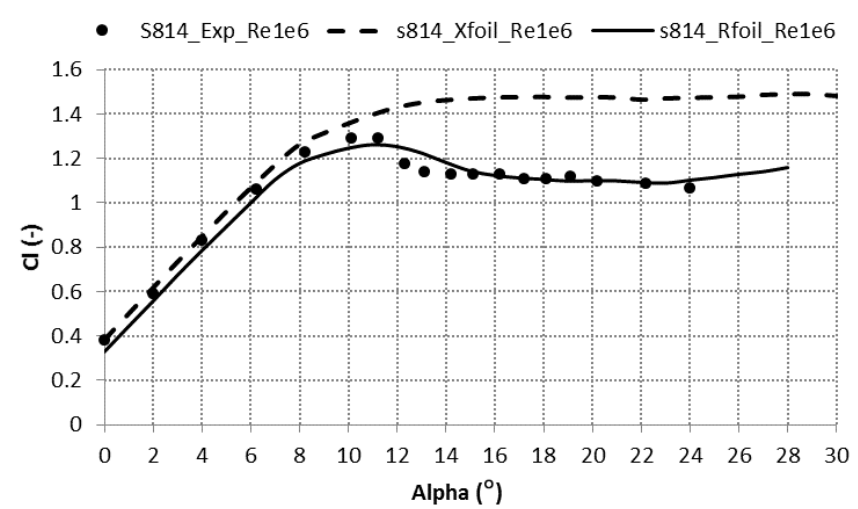

Figure 2. Lift curve for the S814 airfoil. Numerical experimental comparison. Reynolds number: 1 million; free transition.

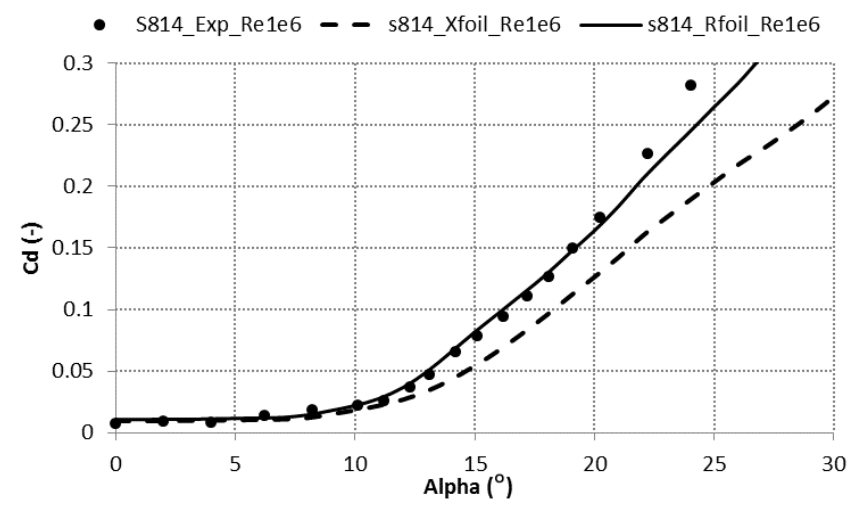

Figure 3. Drag curve for the S814 airfoil. Numerical experimental comparison. Reynolds number: 1 million; free transition.

(Zhou et al., 1999) has been preferred to control the design procedure, where the popular tool RFOIL (van Rooij, 1996) is used to evaluate the aerodynamic performance of the airfoil.

RFOIL is a modified version of XFOIL (Drela, 1989) featuring an improved prediction around the maximum lift coefficient and capabilities of predicting the effect of rotation on airfoil characteristics. In fact, numerical stability improvement is obtained by using the Schlichting velocity profiles for the turbulent boundary layer instead of the Swafford velocity profiles (Schlichting and Gersten, 2017). Furthermore, the shear lag coefficient in Green's lag entrainment equation of the turbulent boundary-layer model is adjusted, and the deviation from the equilibrium flow is coupled to the shape factor of the boundary layer.

Figures 2 and 3 show a comparison between the two codes against S814 airfoil (Somers and Tangler, 1997) wind tunnel data (Somers and Tangler, 1994). As can be observed, RFOIL accuracy for the stall region is significantly better than XFOIL, and, as mentioned in the previous chapters, stall is quite a crucial parameter in this case. Additional validation tests can be found in Grasso (2011) and van Rooij (1996).

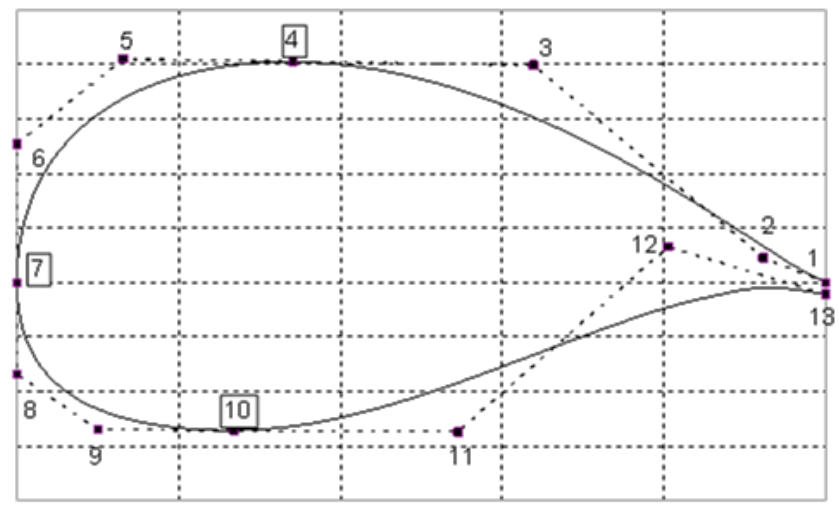

Figure 4. Airfoil shape parameterisation scheme. From Grasso, 2008.

The geometry of the airfoil is parameterised (Grasso, 2008) with a combination of four Bezier curves (see Prautzsch et al., 2002, Barsky, 199, and Beach, 1991, for general information about Bezier curves) of third order distributed along the airfoil contour (Fig. 4). Each Bezier curve covers one quarter of the shape, with 13 control points free to move in chord and normal-to-the-chord directions (i.e. 26 design variables). To appreciate and understand the choice of four Bezier curves, the reader should consider that a thirdorder polynomial is needed to describe inflection points; however, a higher degree can lead to wavy shapes. Dividing the airfoil contour into four pieces is a smart move to divide the complexity of the parameterisation and ease the control of the shape. This formulation is $\mathrm{C}^{2}$ continuous. Overall, 15 design variables are active in the present work; in fact, the leading edge cannot move, while the neighbours and the trailing edge can move only in the vertical direction. In addition, the control points 4 and 10 are internally controlled to ensure $\mathrm{C}^{2}$ property also in those points. The complete mathematical formulation can be found in Grasso (2008).

\section{Results}

\subsection{Airfoil performance}

The blade in development has only two airfoils (one main and one in the inner part, excluding the blending area at the very root of the rotor) in order to simplify the blade construction. The first one is a $30 \%$ thickness airfoil which is used at the maximum chord station, while the second one is a $25 \%$ thickness airfoil which extends from half of the blade span to the tip. A blending area connects these two airfoils. This work focuses on the main airfoil design where the main target is the aerodynamic efficiency $(L / D)$ maximisation at the operative $R e$ of 1 million. At the same time, appropriate stall behaviour needs to be achieved in order to provide good control for the wind turbine, while minimising stall-induced vibrations. 


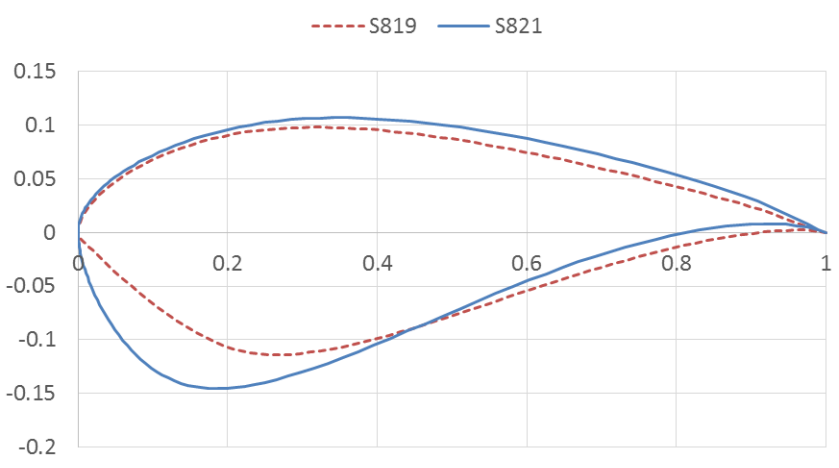

Figure 5. S819 and S821 shapes.

As already mentioned, this aspect plays a crucial role in the present work. From an optimisation point of view, several options in terms of constraints and design points to be included are possible. Some of them are discussed here. High lift performance may lead to sharp stall behaviour; a constraint limiting the maximum lift coefficient can be quite a natural choice. However, limiting the lift coefficient at a specific angle of attack may not be sufficient since there will be no control on different angles. The risk would then be that the stall angle could be delayed or occur earlier, making the constraint (technically satisfied) completely ineffective. The same constraint could be then assigned simultaneously for several angles of attack around the expected stall angle range. This will gain little more confidence but it will add complexity to the optimisation problem and increase the computational costs. Even more dangerous, the risk of limiting the design space too much and driving the solution to local optima would increase. Anyway there will still not be any guarantee about post-stall characteristics, which would still require specific constraint(s). A better and more accurate approach could be to evaluate the full polar at each design iteration and retrieve the information about the maximum lift coefficient and post-stall (via, for instance, the lift slope value). In this way, the number of constraints will reduce to just two, which would fully describe the stall behaviour while keeping the mathematical complexity of the optimisation problem low. However, the computational time would rise because the full polar needs to be calculated for any iteration. On top of that, the same approach should be used in rough conditions to make sure that the airfoil has comparable characteristics in both cases.

Although the latest approach would be the most accurate, a different and more practical solution has been adopted in the present work, which should still have a good level of accuracy. A combination of constraints focused on the maximum lift coefficient $(<1.4)$ and moment coefficient $(>-0.12)$ has been prescribed. In fact both constraints act on the shape of the lift curve bounding its maximum point and its average position in lift axis (i.e. defining the alpha zero lift or the lift at zero degrees), respectively. Considering the airfoil geom-

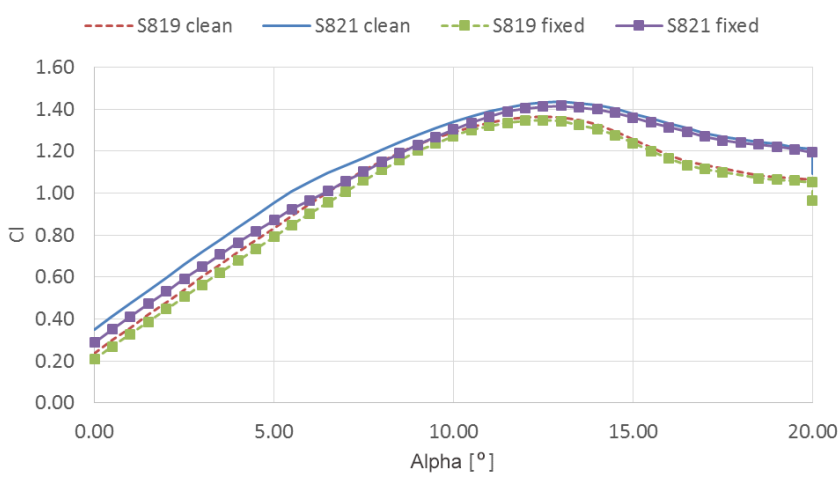

Figure 6. Lift curves for S819 and S821 airfoils. Free- and fixedtransition data; $R e: 1$ million. RFOIL predictions.

etry, both constraints have a direct impact on the camber line of the airfoil and their combined effect is to get soft stall with no excessive cambered shape. Since the roughness generally has little influence on the linear region of the moment coefficient curve, the same constraint on clean conditions should also cover the rough condition.

The airfoil thickness $(t / c)$ of 0.25 has been selected, rather than a thinner value. Although the pure aerodynamic performance could be better with thinner (e.g. $t / c 0.15,0.18$ ) airfoils, thicker sections offer the advantages of saving blade mass and provide higher strength to the blade structure.

Considering existing airfoils, the S821 and the S819 have been used as a reference (Somers, 1993; Tangler et al., 1995; Somers, 1998) because of their good characteristics in terms of insensitivity to roughness and post-stall behaviour. Figure 5 shows the shapes, while Figs. $6-8$ show the aerodynamic performance of these airfoils in free and fixed transition, as calculated with the RFOIL code. The Reynolds number used for the simulations is 1 million, in accordance with the average real Reynolds number value expected for a $60 \mathrm{~kW}$ range machine. All the simulations in fixed transitions (representative of rough condition) presented in this work prescribe a transition at $5 \%$ of the chord on the suction side and $10 \%$ of the chord on the pressure side. It should be noted that the stall and post-stall behaviour is soft but monotonically decreasing in the indicated angle of attack range. In addition, it should be noted that there is a relatively small margin between the design point and the stall; for stall-regulated turbines, this is an important feature to avoid excessive loads once the design condition has been passed (e.g. in case of wind gust).

So the ideal airfoil is a $25 \%$ thick shape (similar to the S821, which is $24 \%$ thick) with an $L / D$ performance similar to $S 819$, a reduced stall margin and a maximum lift coefficient $\left(\mathrm{Cl}_{\max }\right)$, but also a slight roughness sensitivity and a contained moment coefficient $\left(\mathrm{C}_{\mathrm{m}}\right)$; the latter to avoid excessive torsional loads.

With these parameters in mind, three airfoils have been developed to offer better performance than the reference ge- 


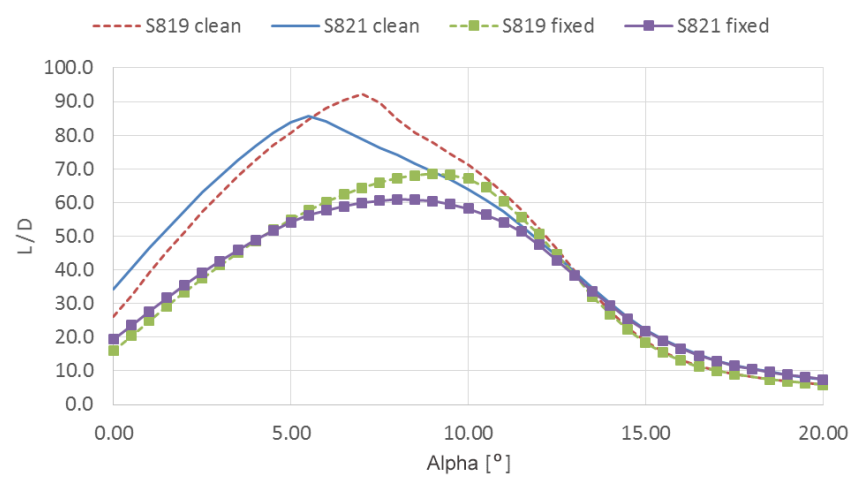

Figure 7. Aerodynamic efficiency curves for S819 and S821 airfoils. Free- and fixed-transition data; Re: 1 million. RFOIL predictions.

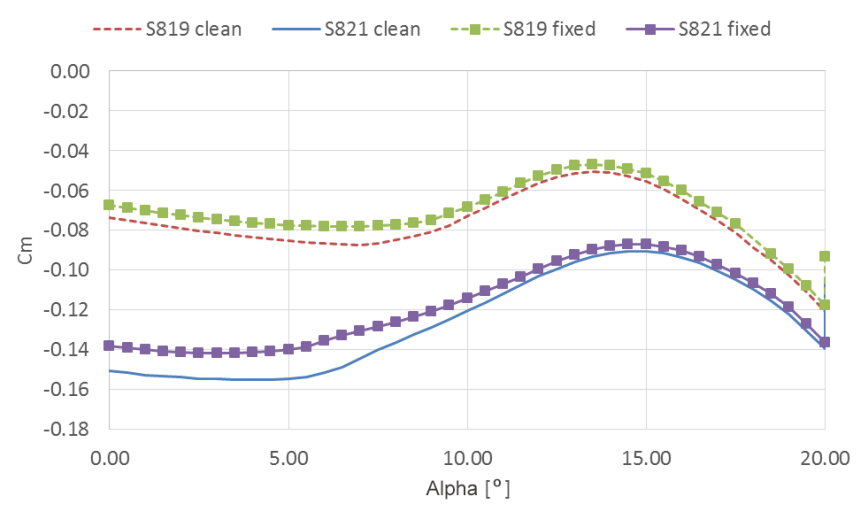

Figure 8. Moment coefficient for S819 and S821 airfoils. Free- and fixed-transition data; $R e$ : 1 million. RFOIL predictions.

ometries. The airfoils have been preliminarily named A, B and $\mathrm{C}$ and are all $25 \%$ thick (the shapes are not shown because of confidentiality issues). Their aerodynamic characteristics, evaluated with RFOIL, are illustrated in Figs. 9 and 10.

The airfoil A has more camber than the other airfoils since the constraint on the moment coefficient discussed above has not been used in order to check the validity of the assumption. This is evident from the lift curve. It achieves better efficiency in clean conditions. However, its behaviour is very sensitive to the roughness; in fixed transition the efficiency drops significantly and the lift curve changes completely, making the control of the wind turbine impossible. The differences are smaller for the airfoil B, but the post-stall characteristics of the lift curve make the control of the turbine difficult. The airfoil C (from now on, called G25sx6) is instead a good compromise between good performance and good control properties. The lift curve is in practice almost unchanged from free to fixed transition, as result of adopting the constraint on the moment coefficient and lift coefficient. In addition, the stall angle of attack is unchanged. In terms of efficiency, the G25sx6 exhibits the best perfor-

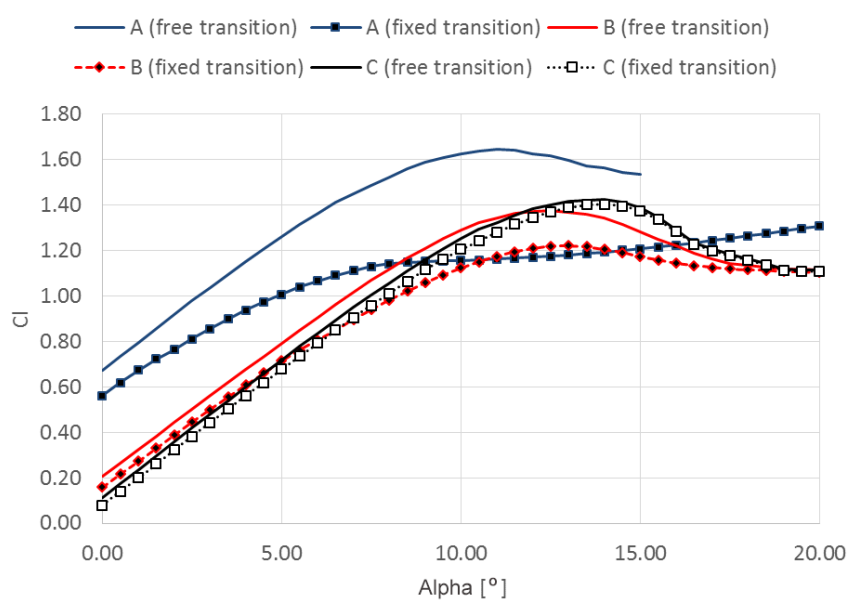

Figure 9. Lift curve of the new airfoils. Free- and fixed-transition data; $R e: 1$ million. RFOIL predictions.

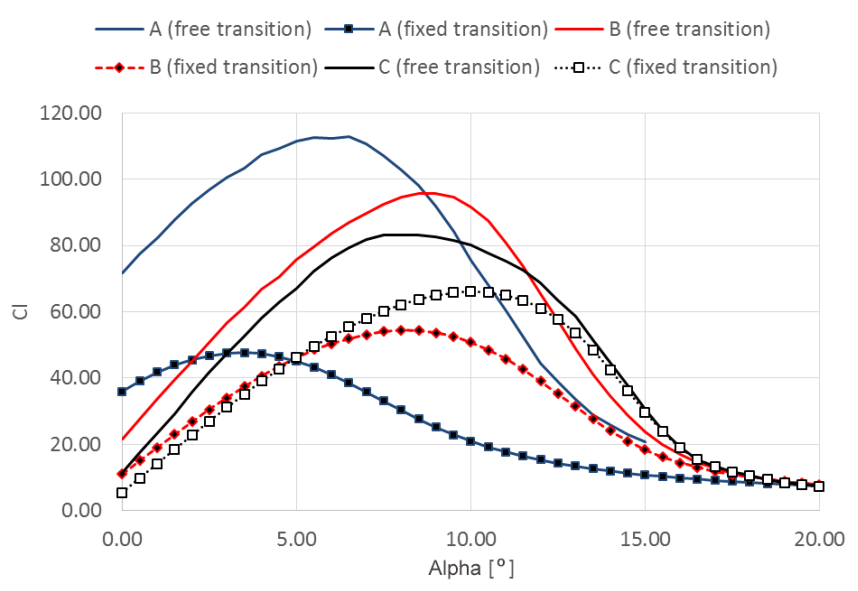

Figure 10. Aerodynamic efficiency curve of the new airfoils. Freeand fixed-transition data; Re: 1 million. RFOIL predictions.

mance in fixed transition and quite a flat plateau in both free and fixed transition. As mentioned, this is quite convenient for stall-regulated turbines because the airfoil will operate in a range of angles of attack rather than a specific value like in the pitch-controlled machines. Combining lift and efficiency performance, the stall margin is almost unchanged between free and fixed transition.

Comparing the G25sx6 with the S821 airfoil (Figs. 11 and 12) a similar value of efficiency in free transition can be seen, but better performance in fixed transition despite the G25sx6 being thicker $(25 \%)$ than the S821 (24\%) is observed.

In addition, the efficiency curves keep a good level over a wider range of angles of attack, and the stall margin is reduced, which is an advantage for stall-regulated wind turbines (i.e. avoiding excessive loads in case of wind gust). 


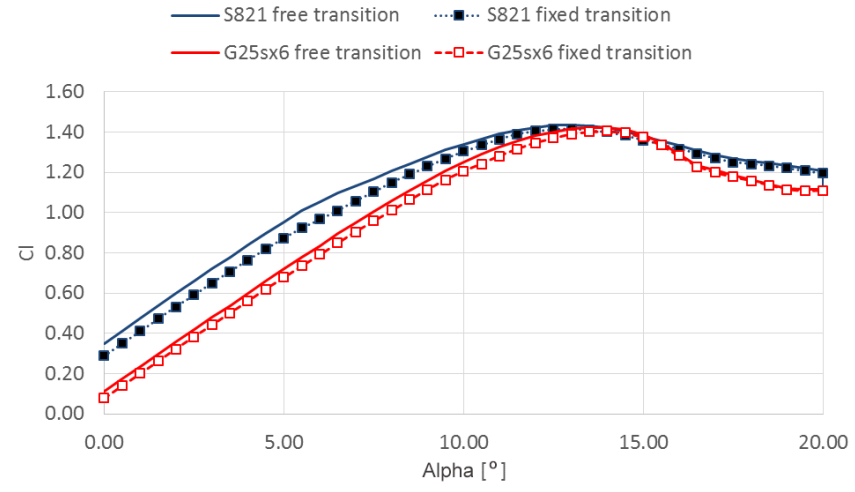

Figure 11. Lift curve of the new airfoil. Free- and fixed-transition data; Re: 1 million. RFOIL predictions.

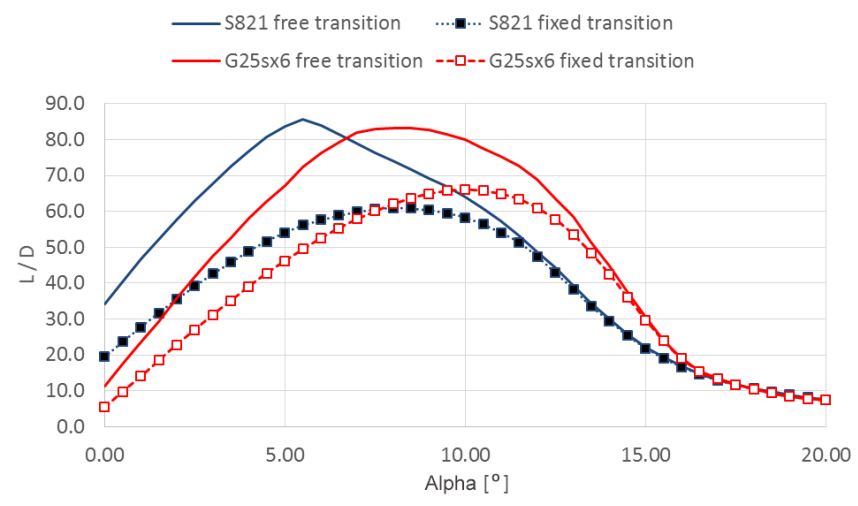

Figure 12. Aerodynamic efficiency curve of the new airfoil. Freeand fixed-transition data; $R e: 1$ million. RFOIL predictions.

\subsection{Optimisation process details}

This section presents some of the details of the optimisation process for the G25sx6 airfoil. As mentioned in the previous paragraph, the $L / D$ was used as a parameter to be maximised. To obtain good roughness robustness, the design has been performed in fixed-transition conditions; in addition, the $L / D$ value was divided by a factor 10 to have the same order of magnitude $(o 1)$ used for the constraints. Figure 13 shows the evolution of the objective function during the iterations of the optimisation process. As can be observed, the trend is not monotonically increasing as one might expect. This is because, to reduce the risk of obtaining a local optimal solution, the NACA0012 airfoil has been used as an initial solution, which is outside the feasible domain $(t / c$ violating the threshold value) and so far from any possible feasible local optima. The optimisation algorithm is designed to obtain first a feasible solution (if any) and then optimise it inside the domain space. Roughly the first 100 iterations are used to obtain a feasible solution. This is evident by looking at Fig. 14, where the evolution of the constraints is illustrated, together with their threshold values identified by the division between the feasible domain (blue area) and the unfeasible

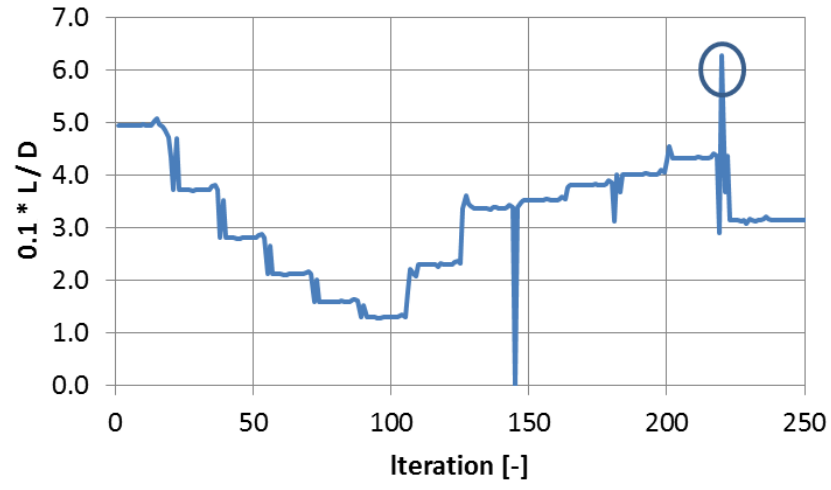

Figure 13. Evolution of the objective function during the design process. Optimal solution highlighted by the circle.

one (red area). The circle in Fig. 13 corresponds to the optimal solution.

\subsection{Impact on rotor performance}

In order to assess the value of the new airfoil, its impact on wind turbine performance has been evaluated with a numerical analysis. This step is important to give a complete overview of the new airfoil effects but is actually necessary to make sure that the optimisation problem has been correctly set up and the constraints are effective in preventing or limiting stall-induced vibration since only the airfoil side of the problem has been investigated after being separated from the rotor response.

A $60 \mathrm{~kW}$ stall-regulated wind turbine has been used as reference and the S821 and G25sx6 airfoils have been adopted as the main airfoil. The reference wind turbine is a threeblade machine designed to produce energy at sites characterised by a very low mean wind speed $\left(4 \mathrm{~m} \mathrm{~s}^{-1}\right)$. Thus, its main characteristics are very low values of cut in and peak power wind speeds (about 2.5 and $8.5 \mathrm{~m} \mathrm{~s}^{-1}$, respectively) and a high AEP with a mean wind speed of about $4 \mathrm{~m} \mathrm{~s}^{-1}$. To obtain this performance a generous rotor radius and particularly slender blades are adopted: the radius is $14 \mathrm{~m}$ and the rotational speed is a constant $34 \mathrm{rpm}$.

Figure 16 shows the power curves for the blade optimised based on the S821 airfoil and G25sx6 airfoil. The blade element momentum (BEM)-based (Hansen, 2007) tool WT_Perf (Buhl, 2004a, b) developed by the National Renewable Energy Laboratory (NREL) has been used for these analyses.

The blade geometry has been adjusted to consider the actual airfoils adopted. Normally, this includes chord and twist; however, in this case, the same chord distribution has been used (Fig. 15) since preliminary analyses showed little impact on overall performance.

As already mentioned, the G25sx6 is $1 \%$ thicker than the S821; this ensures a higher moment of inertia of each section implying a lower weight of the blade. From a prelim- 

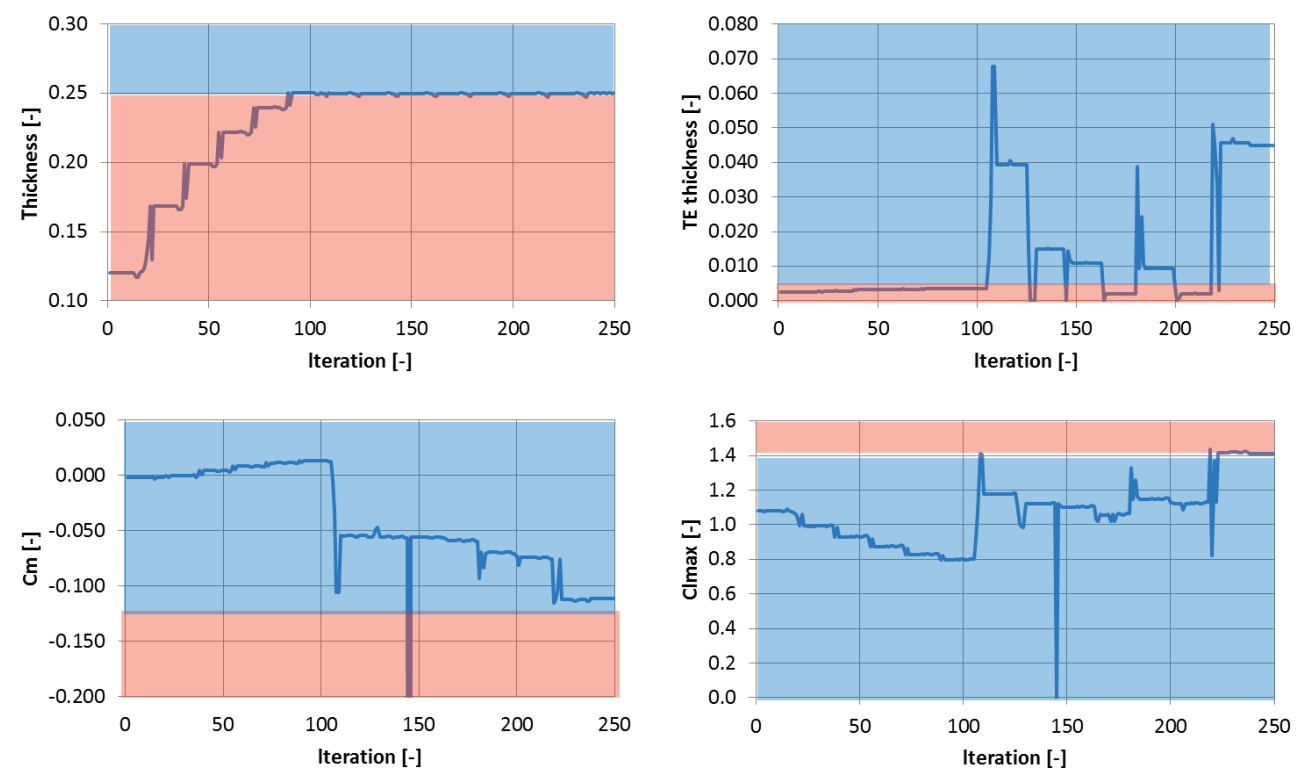

Figure 14. Evolution of the constraints during the design process. The blue region corresponds to the feasible domain, while the red one corresponds to the unfeasible domain.

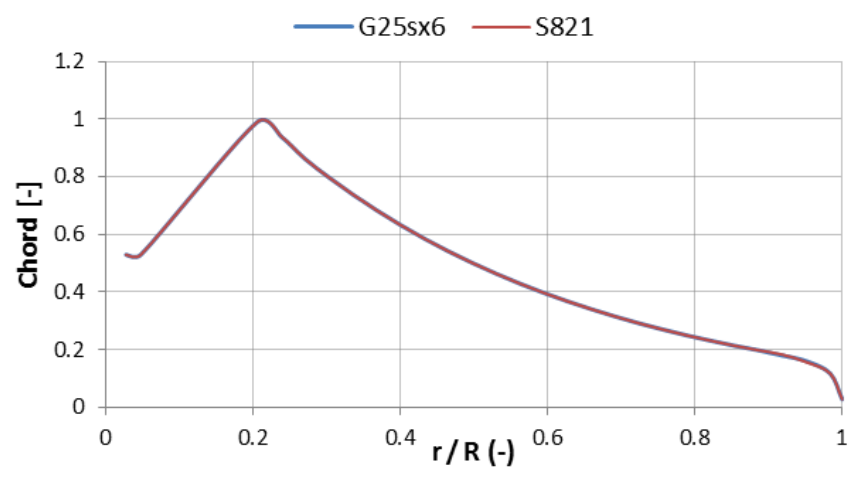

Figure 15. Chord distribution adopted during the blade design.

inary analysis, the weight of the blade can be reduced by about $5 \%$.

Both free- and fixed-transition conditions have been included, as representative of clean and rough blade conditions. According to the results, there are no symptoms of stallinduced vibration. This was not expected to happen anyway, but since the airfoil design has been performed in fixed transition, the performance in clean conditions could have been subject to the risk of stall-induced vibration.

The power curves related to free and fixed transition in Fig. 16 refer to different values of the blade pitch, which is the value necessary to achieve the desired peak power in each case.

Since in fixed transition the lift coefficient (particularly the maximum lift coefficient) is lower than in free transition, a larger pitch angle will be necessary to reach the desired peak

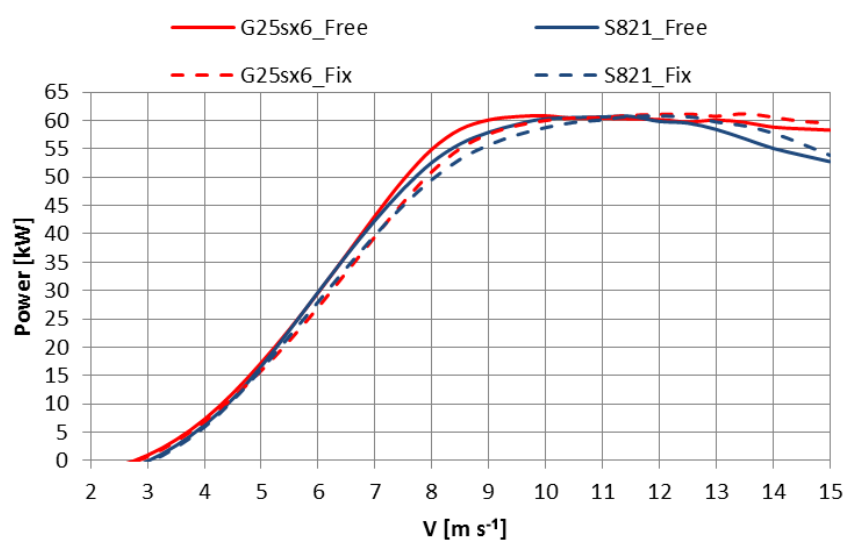

Figure 16. Effect of the new airfoil on the wind turbine power curve.

power. At the same time, a higher wind speed is needed to reach the same peak power.

Figure 17 shows the angle of attack distribution along the blade at $5 \mathrm{~m} \mathrm{~s}^{-1}$ and in free-transition conditions for both the wind turbines. The unusual distribution that can be seen at the tip of the blade is due to the twist distribution adopted to reduce stall-induced vibrations, reduce the loads and improve the overall stability; this feature, together with the rest of the blade design strategy and process, will be discussed in a dedicated work.

Considering the overall AEP (see Table 1), the new airfoil provides a considerable gain in free $(+5.1 \%)$ and fixed $(+2.3 \%)$ conditions. In more detail, the turbine reaches its maximum power for lower wind speed and the post-peak re- 


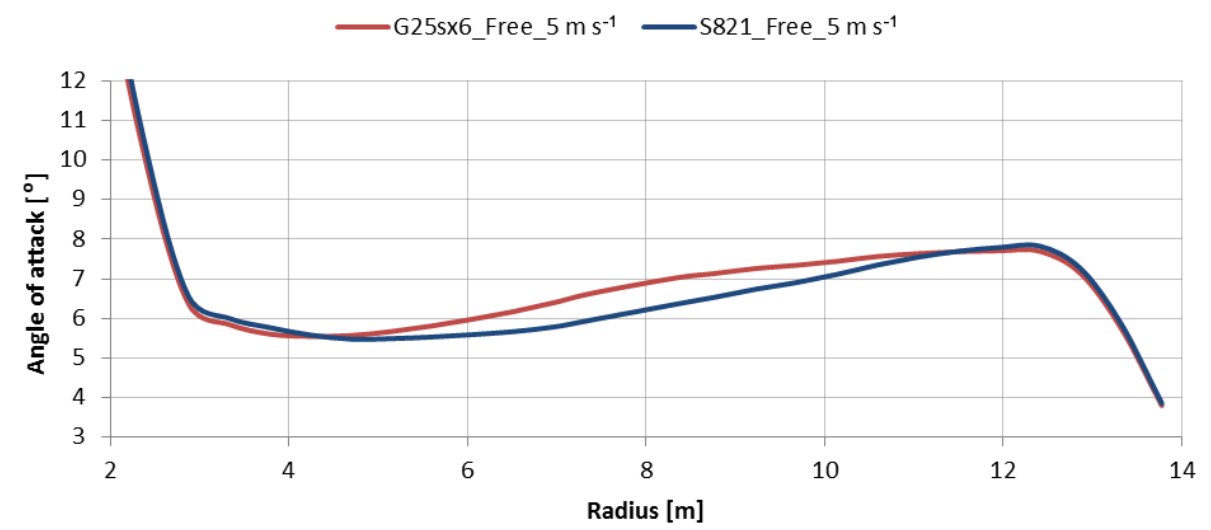

Figure 17. Angle of attack distribution along the blade.

Table 1. Impact of the new airfoil on the wind turbine AEP.

\begin{tabular}{lrrrr}
\hline Airfoil & \multicolumn{2}{c}{ Free transition } & \multicolumn{2}{c}{ Fixed transition } \\
\cline { 2 - 5 } & AEP $(\mathrm{kWh})$ & $\Delta(\%)$ & AEP $(\mathrm{kWh})$ & $\Delta(\%)$ \\
\hline S821 & 136000 & - & 129000 & - \\
G25sx6 & 143000 & +5.15 & 132000 & +2.3 \\
\hline
\end{tabular}

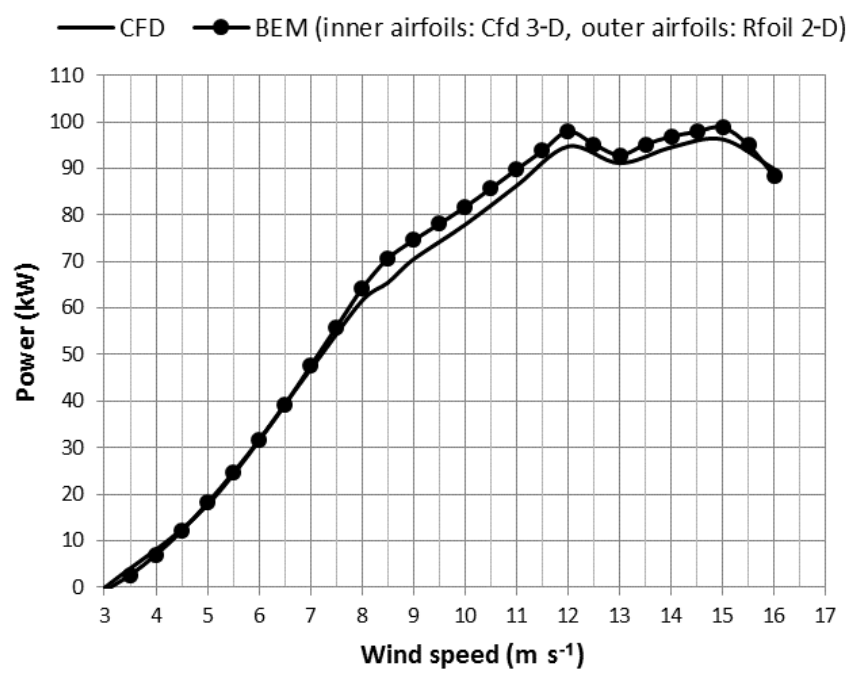

Figure 18. CFD power curve vs. Wt_Perf power curve implementing aerodynamic curves of inner airfoils extracted from CFD.

gion is smoother. In addition, the production at very low wind speed increases thanks to the new airfoils.

\subsection{Verification of WT_Perf for rotational effects}

The findings illustrated so far are based on BEM assumptions and WT_Perf accuracy. In particular, the flow at the root is a critical point. In fact, lift and drag coefficients of root airfoils of a rotating blade are affected by the so-called "stall delay" phenomenon (Himmelskamp, 1947; Guntur, 2011; Herráez, 2014); so the two-dimensional aerodynamic curves of these airfoils need to be adjusted at high angles of attack before being used in a BEM code like Wt_Perf to consider rotational effects. In this work, lift and drag coefficients of the inner airfoils (approximately from root to $20 \%$ of the blade) have been extrapolated from a computational fluid dynamics (CFD) analysis of a rotating blade following the inverse BEM method reported in Guntu and Słrensen (2014), while two-dimensional aerodynamic coefficients obtained by using RFOIL have been used for the airfoils along the outer half of the blade, where rotational effects can be neglected (Tangler, 2005). This method is useful to speed-up the wind turbine optimisation process because it allows us to modify the outer part of the blade, which is most influential for the performances and behaviour of the whole system, simply by using two-dimensional aerodynamic airfoil characteristics.

One of the preliminary blades designed during this work has been used as a reference to validate the method. Despite the fact that the design was intended to produce a $60 \mathrm{~kW}$ machine, the actual results ended in a rejected design since it failed to be controllable. This fact, however, made the design an interesting test case for validation because of two distinct peaks in the power curve. Figure 18 shows the comparison between the power curve predicted with CFD analysis in steady operating conditions and the power curve obtained with the method used in this work. STAR-CCM software has been used, with the $k \omega$ turbulence model. As can be seen, the agreement is very good, as the BEM-based scheme captures not only the general trend but also the two peaks at 12 and $15 \mathrm{~m} \mathrm{~s}^{-1}$ wind speed. A publication dedicated to the topic is under preparation at the moment which will provide the full details on the development done on the subject. 


\section{Conclusions}

Despite the fact that the pitch-controlled wind turbines cover the complete large-megawatt machines market, stallregulated solutions are still diffused for small power production. A new airfoil specifically designed for this class of wind turbines has been developed and presented in this work. Compared to existing geometries, the new airfoil can increase the annual energy production of the machine, both in clean and rough conditions. In terms of rotor performance, the new airfoil brings an evident benefit to the punctual power production and to the overall AEP $(+5.1 \%$ in free transition and $+2.3 \%$ in fixed transition).

Data availability. The data are not publicly available as they refer to the characteristics/performance of a new industrial product which is currently under development and the data are therefore confidential. The mathematical models used in the present work are thirdparty tools and can be accessed via the following links: RFOIL, www.ecn.nl; XFOIL, http://web.mit.edu/drela/Public/web/xfoil/; WT_perf, https://github.com/NREL/HARP_Opt/tree/ master/Source/WT_Perf/v3.05.00a-adp; http://www2.cd-adapco.com/1/14592/2013-07-10/5118m.

Competing interests. The authors declare that they have no conflict of interest.

Special issue statement. This article is part of the special issue "The Science of Making Torque from Wind (TORQUE) 2016". It is a result of the The Science of Making Torque from Wind (TORQUE 2016), Munich, Germany, 5-7 October 2016.

Edited by: Carlo L. Bottasso

Reviewed by: two anonymous referees

\section{References}

Barsky, B. A.: Acm/siggraph '90 course 25: Parametric bernstein/bezier curves and tensor product surfaces, Dallas, TX, 1990.

Beach, B. C.: An Introduction to Curves and Surfaces of ComputerAided Design, Van Nostrand Reinhold, 1991.

Bizzarrini, N., Grasso, F., and Coiro, D. P.: Genetic Algorithms in Wind Turbine Airfoil Design, Proceedings of EWEA2011, Bruxelles, March 2011.

Buhl Jr., M. L.: A New Empirical Relationship between Thrust Coefficient and Induction Factor for the Turbulent Windmill State. NREL/TP-500-36834, Golden, CO, National Renewable Energy Laboratory, September, 2004a.

Buhl, M. L.: WT_perf user's guide, National Wind Technology Centre, NREL, Golden, Co, USA, 2004b.

Drela, M.: XFOIL: An Analysis and Design System for Low Reynolds Number Airfoils, Conference on Low Reynolds Number Airfoil Aerodynamics, Lecture Notes in Engineering, Uni- versity of Notre Dame, 54, 1-12, https://doi.org/10.1007/978-3642-84010-4, 1989.

Fletcher, R.: Practical Methods of Optimization, Wiley, ISBN: 9780471494638, 1987.

Grasso, F.: Multi-Objective Numerical Optimization Applied to Aircraft Design, Ph.D. Thesis, Dip. Ingegneria Aerospaziale, Università di Napoli Federico II, Napoli, Italy, 2008.

Grasso, F.: Usage of Numerical Optimization in Wind Turbine Airfoil Design, AIAA, J. Aircraft, 48, 248-255, https://doi.org/10.2514/1.C031089, 2011.

Grasso, F.: Hybrid Optimization of Wind Turbine Thick Airfoils, Proceedings from 9th AIAA Multidisciplinary Design Optimization Specialists Conference, Honolulu, AIAA 2012-1354, https://doi.org/10.2514/6.2012-1354, 2012.

Guntur, S. and Sørensen, N. N.: An evaluation of several methods of determining the local angle of attack on wind turbine blades, J. Phys. Conf. Ser., 555, 012045, https://doi.org/10.1088/17426596/555/1/012045, 2014.

Guntur, S., Bak, C., and Sørensen, N.: Analysis of 3D stall models for wind turbine blades using data from the MEXICO experiment, in: Proceedings of the 13th International Conference on Wind Engineering, Amsterdam, the Netherlands, 10-15 July 2011.

Hansen, M. O.: Aerodynamics of wind Turbines, 2nd Edn., Routledge, December 2007, ISBN: 978-1844074389, 2007.

Herráez, I., Stoevesandt, B., and Peinke, J.: Insight into Rotational Effects on a Wind Turbine Blade Using Navier-Stokes Computations, Energies, 7, 6798-6822, https://doi.org/10.3390/en7106798;ISSN 1996-1073, 2014.

Himmelskamp, H.: Profile Investigations on a Rotating Airscrew, Reports and Translations/MAP Völenrode, 1947.

Petersen, J. P., Madsen, H. A., Bjork, A., Enevoldsen, P., Oye, S., Ganander, H., and Winkelaar, D.: Predictions of Dynamic Loads and Induced Vibration in Stall, Risoe, Risoe-R-1045, May, 1998.

Prautzsch, H., Boehm, W., and Paluszny, M.: Bezir and B-Spline Techniques, Springer, ISBN: 978-3540437611, 2002.

Rasmussen, F.: Dynamic stall of a Wind Turbine Blade Section, Proceedings of the 8th IEA Joint Action Symposium on Aerodynamics of Wind Turbines, 1994.

Rasmussen, F., Petersen, J. T., Winkelaar, D., and Rawlinson-Smith, R.: Response of Stall Regulated Wind Turbines - Stall Induced Vibrations, final report for Joule I Project, Contract no. JOUR0076, DG XII, Report Riso-R-691 (EN), Risoe, June 1993.

Somers, D. M.: The S819, S820 and S821 Airfoils, NREL, NREL/SR-500-36334, October 1992-November 1993, 1993.

Somers, D. M.: Effects of Airfoil Thickness and Maximum Lift Coefficient on Roughness Sensitivity, Airfoils, Inc., 1998.

Somers, D. M. and Tangler, J. L.: Wind Tunnel Test of the S814 Thick Root Airfoil, ASME, J. Sol. Energ.-T. Asme, 118, 217221, https://doi.org/10.1115/1.2871781, 1994.

Somers, D. M. and Tangler, J. L.: Design and experimental results for the S814 airfoil, NREL/SR-440-6919, 1997.

Schlichting, H. and Gersten, K.: Boundary Layer Theory, 9th Edn., Springer, ISSBN 978-3662529171, 2017.

Tangler, J. D. and Kocurek, J.: Wind Turbine Post-Stall Airfoil Performance Characteristics Guidelines for Blade-Element Momentum Methods, Report NREL/CP-500-36900, 2005.

Tangler, J. L. and Somers, D. M.: NREL Airfoil Families for HAWTs, NREL/TP-442-7109, 1995. 
van Rooij, R. P. J. O. M.: Modification of the boundary layer calculation in RFOIL for improved airfoil stall prediction, Report IW-96087R TU-Delft, the Netherlands, September 1996.

Zhou, J. L., Tits, A. L., and Lawrence, C. T.: User's guide for FFSQP version 3.7: A FORTRAN code for solving constrained nonlinear optimization problems, generating iterates satisfying all inequality and linear constraints, University of Maryland, College Park, 1999. 